\title{
Translational Medicine Communications: the home for sound translational science
}

\author{
Francesco Marincola ${ }^{1}$ and Philip Dooner ${ }^{2^{*}}$
}

\section{Introduction}

We are delighted to announce the launch of Translational Medicine Communications (TMC), a companion journal to Journal of Translational Medicine [1]. With the National Center for Advancing Translational Sciences (NCATS) currently developing its first strategic plan to "identify critical challenges, compelling opportunities, and emerging and unmet needs; develop and set scientific and operational goals and research priorities; and engage a diverse and broad community of stakeholders" [2], the time has never been better to launch a journal that will be a home for the translational sciences community.

The open access nature of TMC has many advantages including free and universal online access to the article immediately upon publication, giving it the potential to reach a much larger audience than any subscriptionbased journal. Copyright is also retained by the author.

Being an online only journal, there are also no page or color charges and we can host unlimited numbers of figures, data sets and movies. A single Article Processing Charge (APC) is applied to all articles, regardless of their length and we aim to ensure that the APC is always competitive and affordable.

\section{Scope}

$T M C$ aims to publish open access research, as well as review, methodology and protocol articles that facilitate the communication between basic and clinical science. Like Journal of Translational Medicine, TMC covers all areas of translational medicine and retains its specialised sections.

$T M C$ also believes that all scientifically valid research deserves to be published and made accessible to the translational research community.

\footnotetext{
* Correspondence: philip.dooner@biomedcentral.com

${ }^{2}$ BioMed Central, London, UK

Full list of author information is available at the end of the article
}

\section{Relationship with Journal of Translational Medicine}

As mentioned previously, $T M C$ is the companion journal to Journal of Translational Medicine and shares a similar scope and many of the same Editorial Board Members. However, unlike Journal of Translational Medicine, which aims to publish articles contributing to the significant advancement of translational science, $T M C$ only assesses a manuscript's suitability for publication based on its scientific validity.

Because TMC is led by the same expert editorial team as Journal of Translational Medicine, it means that manuscripts submitted to Journal of Translational Medicine, but not of sufficient advance to warrant publication, may be deemed suitable for publication in TMC prior to submission. In these instances, it is possible to transfer the manuscript files and any reviewer comments automatically and the Editor should be able to make a decision, or give guidance about revision, without sending the manuscript out for another round of review.

\section{Peer review}

TMC uses the same team of specialised Section Editors, each one an expert in their own field.

The journal aims to provide authors with a first decision on their manuscript within six weeks. Upon submission, manuscripts are assigned to one of the Section Editors, or one of the journal's knowledgeable Deputy Editors, Senior Editors or Associate Editors. Each handling editor will critically assess the manuscript, and when appropriate provide necessary detailed input. The manuscript will also be assessed by two independent referees as standard.

The aim of the peer review process is to establish the soundness of a manuscript. Referees and Editors will determine whether a paper is scientifically valid, rather than making judgements on interest levels or whether the submission represents a significant advance.

The journal editors will try and keep the number of major revision requests to a minimum, and limit requesting unnecessary further experiments. 


\section{Competing interests}

The authors have no competing interests.

\section{Author details}

${ }^{1}$ Sidra Medical and Research Center, Doha, Qatar. ${ }^{2}$ BioMed Central, London, UK.

Received: 2 August 2016 Accepted: 5 August 2016

Published online: 30 August 2016

\section{References}

1. J Transl Med: http://translational-medicine.biomedcentral.com/. Accessed 20 June 2016

2. NCATS Strategic Plan: https://ncats.nih.gov/strategicplan. Accessed 20 June 2016

Submit your next manuscript to BioMed Central and we will help you at every step:

- We accept pre-submission inquiries

- Our selector tool helps you to find the most relevant journal

- We provide round the clock customer support

- Convenient online submission

- Thorough peer review

- Inclusion in PubMed and all major indexing services

- Maximum visibility for your research

Submit your manuscript at www.biomedcentral.com/submit 